\title{
Pulmonary Resection for Metachronous Lung Cancer Following Contralateral Pneumonectomy
}

\author{
Thomas Tsitsias ${ }^{1}$, Prity Gupta ${ }^{1}$, Kelvin W. Lau ${ }^{1}$, Nicholas Harvey ${ }^{2}$, Sridhar Rathinam ${ }^{1}$ \\ ${ }^{1}$ Department of Thoracic Surgery, University Hospitals of Leicester, Leicester, UK; ${ }^{2}$ Department of Cardiothoracic Anesthesia, Uni- \\ versity Hospitals of Leicester, Leicester, UK. \\ Email: tomtsitsias@gmail.com
}

Received December $1^{\text {st }}, 2012$; revised January $5^{\text {th }}, 2013$; accepted January $16^{\text {th }}, 2013$

\begin{abstract}
Patients who undergo pneumonectomy for lung cancer are at risk of recurrent disease and metachronous tumours in the remaining lung. Establishing a diagnosis can be difficult as biopsy may induce a pneumothorax, whilst resection is limited by remaining lung function. However, pneumonectomy should not be a contraindication to further lung resection. We report two cases of lung cancer resection in the residual lung 6 and 11 years following pneumonectomy respectively. A limited resection can be safely performed for a new pulmonary parenchymal lesion on a background of a previous pneumonectomy.
\end{abstract}

Keywords: Metachronous Lung Cancer; Intermittent Apnea; Limited Resection

\section{Introduction}

Patients treated with pulmonary resection for bronchogenic carcinoma still carry the risk of second primary tumour arising from the remaining parenchyma that ranges from $2 \%$ to $5 \%$ every year $[1,2]$. Surgical treatment remains the management of choice for patients who develop a new lung lesion. Only a few decades ago many physicians thought of lung resection on a single remaining lung as an absolute contraindication. The report though of limited case series [3,4] with encouraging results have started to change the clinicians approach to this complicated condition. Unfortunately there is still a considerable number of patients with adequate functional reserve and no distal metastases that have never been referred, nor even considered for resection being deprived in that way of a potentially lifesaving therapeutic option.

We report two separate cases managed in our Department with curative thoracoscopic pulmonary resections 11 and 6 years after contralateral pneumonectomy, respectively.

\section{Case Reports}

\subsection{Case 1}

A 67-year-old male ex-smoker with a 60 pack years smoking history presented to emergency department with right sided chest pain, 11 years after a left pneumonec-tomy for a pT2aN1 (7th edition Lung Cancer stag- ing, American Joint Committee on Cancer) large cell undifferentiated tumor followed by adjuvant chemotherapy. Physical examination was unremarkable apart from tenderness on palpation over the right sided chest. Imaging by means of a CT scan was performed and revealed a $26 \times 21 \mathrm{~mm}$ speculated right upper lobe mass (Figure 1(a)). Positron emission tomography (PET) scan showed a maximum standardized uptake value $\left(\mathrm{SUV}_{\max }\right)$ of 6 with no evidence of nodal or distant metastases (Figure 1(b)). His performance status was 1 and generally unaffected by the pain with a forced expiratory volume (FEV1) $1.56 \mathrm{~L}$ (54\% predicted) and transfer factor for carbon monoxide (TLCO) of $66 \%$ predicted. His case was discussed in the Lung multidisciplinary team meeting (MDT) which felt the lesion is a metachronus primary rather than a secondary. Percutaneous CT guided biopsy has the risk of a fatal pneumothorax of the single lung; hence a surgical excision biopsy was the only option. He was counseled and consented for a Video assisted thoracoscopic assessment and resection.

His thoracoscopic approach of this right upper lobe mass was commenced under intermittent apnea. Anaesthetist hyperoxygenated the right lung to achieve saturations of $100 \%$ and then turned the ventilator off to allow the surgeon to proceed to resection. The tumor in the upper lobe was assessed and a limited utility thoracotomy was performed for safe application of stapling device on a position to achieve clear resection margins. The resected lesion demonstrated a T2N0 (invading vis- 


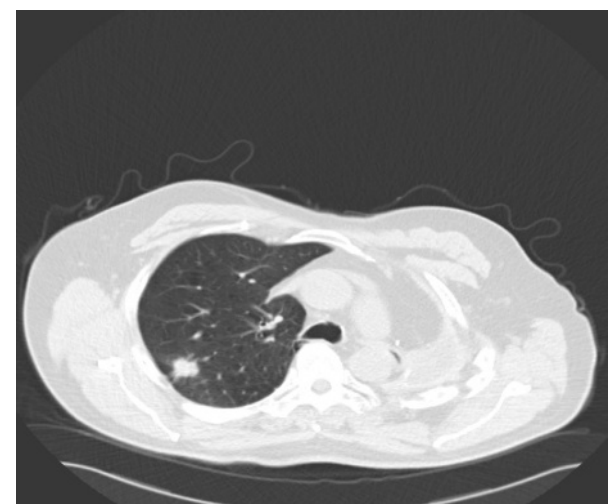

(a)

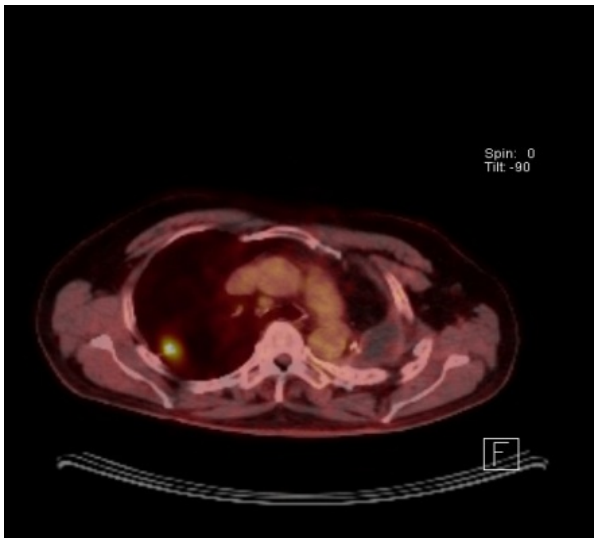

(b)

Figure 1. (a) CT scan showing a speculated mass in the right upper lobe and left pneumonectomy space; (b) PET CT with metabolic activity in the mass $S U V_{\text {max }}: 6$.

ceral pleura, PL2) moderately differentiated adenocarcinoma with clear resections margins. The patient was discharged on day 2 without any complications and has been followed up for 13 months without any evidence of recurrence. No adjuvant chemotherapy was given.

\subsection{Case 2}

A 70-year-old female ex-smoker (100 pack years) was incidentally noted to have a $2.3 \mathrm{~cm}$ cavitating right upper lobe lesion on routine surveillance Computed Tomography scan (Figure 2(a)), 6 years after a left pneumonectomy for a stage IIIb squamous cell carcinoma for which she had received adjuvant chemotherapy. Positron emission tomography (PET) showed an $\mathrm{SUV}_{\max }$ of 2.8, with low uptake at the subcarinal node (Figure 2(b)). She had a performance status of 1 and pre-operative FEV1 0.96 L (45\% predicted) and TLCO 62\% predicted. Prior to lung resection a cervical mediastinoscopy was performed to assess the nodal status and showed that mediastinal nodes were negative for malignancy. Her case was discussed in the Lung MDT with a similar consensus to the previous patient. She was counseled and

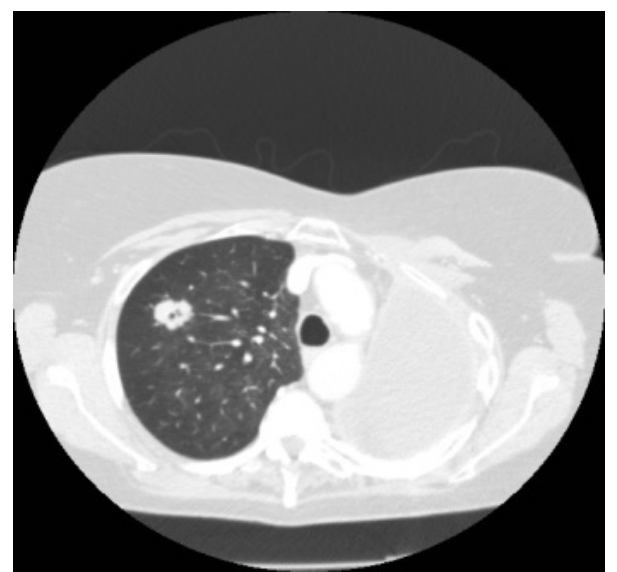

(a)

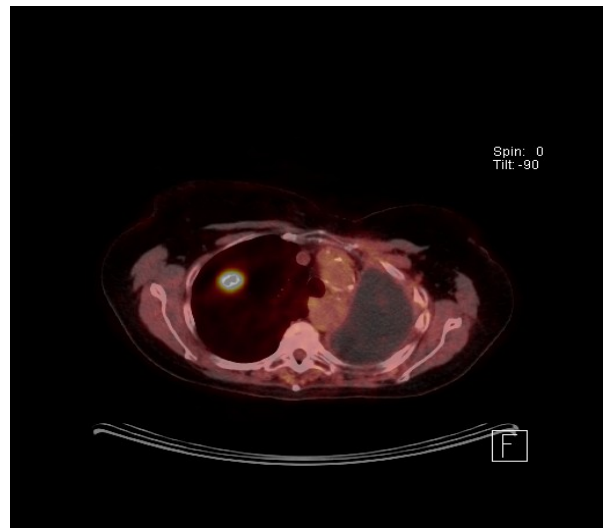

(b)

Figure 2. (a) CT scan showing a speculated mass in the right upper lobe and left pneumonectomy space; (b) PET CT with metabolic activity in the mass $S_{U V} V_{\text {max }}: 2.8$.

consented for VATS assessment and resection.

She underwent a VATS right upper lobe wedge resection with a utility thoracotomy under intermit tent apnea. This confirmed a T1a adenocarcinoma. The patient was discharged on day 5 without any complications and has been followed up for 6 months. No evidence of recurrence has been identified on the follow up surveillance.

\section{Discussion}

The risk of a new lung cancer or metastatic disease following pneumonectomy is well recognized and every clinician involved on the management of such patients should be alert on their follow-up surveillance. There are less than 100 cases in the literature reported during the previous 35 years [5-8]. Factors that have limited surgical intervention on such patients are pulmonary reserve, the presence of distal metastasis but mainly the misconception by many physicians that a previous pneumonictomy is an absolute contraindication for subsequent lung resection. Studies with encouraging results have shown that in carefully selected patients long-term survival with 
very low operative mortality can be achieved [2].

A new primary lung cancer can occur at any time after curative resection at a range of $1 \%$ to $5 \%$ per year. In both of our cases the new lung lesion on the single remaining lung proved to be metachronous lung primary of different histological type than the contralateral lung cancer for that had necessitated a pneumonectomy. No definite criteria to differentiate between a metachronous primary cancer and metastatic disease exist currently. However the vast majority of reported series use the criteria suggested by Martini and Melamed [9] in 1975 according to which a second lung tumour in a patient previously operated on for lung cancer should be considered a second primary (metachronous) and not a metastasis when the cell type of the two lesions is different, the time interval between the two lesions is greater than 2 years, and there are no other distant metastases.

The majority $(70 \%-80 \%)$ of patients with metachronus lung cancer are asymptomatic and the tumour is usually an incidental finding on surveillance chest roentgenograms $[10,11]$. When present the symptoms usually comprise of cough, hemoptysis and dyspnea [1,11]. This shows the importance of systematic and long-term follow-up of patients who undergo successful resection for lung cancer.

The preoperative workup of these patients involves the same tests as diagnosis of the initial cancer. Radiologic evaluation by means of standard chest roentgenogram and thoracic and upper abdomen computed tomography should be performed. Positron emission tomography scan is gradually becoming a standard assessment tool in the oncological evaluation of metachronus lung lesions giving the clinicians the advantage of assessing the mediastinum, becoming a reliable alternative to routine mediastinoscopy. However, if evidence of mediastinal lymphadenopathy is found then nodal sampling by mediastinoscopy should be performed before the resection. Both our patients underwent PET CT scan before the operation that revealed an increased metabolic activity of the speculated lesion.

Establishing the nature of these lesions becomes challenging as a percutaneous CT guided biopsy has the risk of a fatal pneumothorax of the single lung. In the current environment offering curative radiotherapy or chemotherapy in the absence of histological diagnosis is not best practice. Particularly if there is a feeling the lesion is metachronus primary rather than a secondary. Hence a surgical excision biopsy is the only option in a fit patient with good performance status.

Functional evaluation includes standard pulmonary function tests (PFTs) and case series in the past have suggested the use of a stair climbing test (6 MWT) to evaluate cardiopulmonary reserve [3]. A 6MWT of less than $2000 \mathrm{ft}$ (roughly $600 \mathrm{~m}$ ) correlates with a VO2 max of less than $15 \mathrm{ml} / \mathrm{kg} / \mathrm{min}$. In the case series reported by Grodzki et al. [4] all patients had standardized exercise test and echocardiography along with standard PFTs prior to the surgery. Our patients had a preoperative FEV1 of $54 \%$ and $46 \%$ respectively and predicted postoperative FEV1 values that would allow for wedge or segmental resection based on the British Thoracic Society (BTS) guidelines on selection of patients with lung cancer for surgery [12]. According to these the amount of resectable lung volume should be determined by the lung function tests primarily, regardless of the site of pneumonectomy. However, careful correlation and subjective evaluation of the patient's exercise tolerance is of great importance.

Underlying pulmonary function and the patient's performance status preoperatively can determine the extent of pulmonary resection a patient can tolerate as well as the size and location of the tumour. Major resections like lobectomy, segmentectomy or multiple wedge resections may cause concern in such patients. Even though they are occasionally reported [4] they are usually contraindicated since they have been associated with a poor outcome. Lobectomy can be an option when the middle lobe is the one considered for resection [13]. Postoperative respiratory function is dependent on the extent of resection and restrictive effect of the thoracotomy. The manipulation and contusion of residual lung may also impair the already affected pulmonary reserve; therefore resection should be performed using decreased tidal volumes to minimize barotraumas and parenchymal damage related to extensive manipulation of a hyperinflated lung. So every effort must be taken to perform a minimally invasive procedure (VATS). However VATS is challenging as there is no chance of collapsing the lung to perform the surgery, hence the only option is to perform the procedure with intermittent apnea.

The resection of metachronus lung lesions is a challenge for all the members of the team involved on such patients' management. Periods of intermittent apnea after hyperoxygenation have been used by the anesthetic team on both our patients to facilitate deeper wedge excision and ensure that clear surgical margins will be achieved. During the apnoic period oxygen saturations were closely observed with an aim to maintain them above $85 \%$ - 88\%. Other centres have used more interventional techniques like cardiopulmonary bypass (CPB) and high frequency jet ventilator to allow the surgeon to work on a collapsed lung. However it is not recommended as standard approach for these patients from an anesthetic point of view. The use of epidural analgesia has enabled the thoracic teams to deliver effective analgesia, adequate pain control and conesquently expectoration of secretions.

The long-term survival for metachronus tumours has been shown to be better than for metastatic disease in the 
limited case series published so far. In cases that differentiation between the two conditions is difficult and the histology of the new lung lesion cannot be determined prior to surgery, pulmonary resection is warranted. Although the vast majority of resections that will be feasible are going to be wedge resections hence inappropriate for lung cancer, it is widely believed that in these high risk patients, limited resection provides the best riskbenefit ratio [2].

\section{REFERENCES}

[1] C. Deschamps, P. C. Pairolero, V. F. Trastek and W. S. Payne, "Multiple Primary Lung Cancers," The Journal of Thoracic and Cardiovascular Surgery, Vol. 99, No. 5, 1990, pp. 769-778.

[2] T. W. Shields, C. T. Drake and J. C. Sherick, "Bilateral Primary Bronchogenic Carcinoma,” The Journal of Thoracic and Cardiovascular Surgery, Vol. 48, 1964, pp. 401417.

[3] A. Terzi, A. Lonardoni, P. Scanagatta, S. Pergher, C. Bonadiman and F. Calabrò, "Lung Resection for Bronchogenic Carcinoma after Pneumonectomy: A Safe and Worthwhile Procedure," European Journal of Cardio-Thoracic Surgery, Vol. 25, No. 3, 2004, pp. 456-459. doi:10.1016/j.ejcts.2003.12.024

[4] T. Grodzki, J. Alchimowicz, A. Kozak, B. Kubisa, J. Pieróg, J. Wójcik, et al., “Additional Pulmonary Resections after Pneumonectomy: Actual Long-Term Survival and Functional Results," European Journal of Cardio-Thoracic Surgery, Vol. 34, No. 3, 2008, pp. 493-498. doi:10.1016/j.ejcts.2008.05.023

[5] T. A. Salerno, D. D. Munro, P. E. Blundell, et al., "Second Primary Bronchogenic Carcinoma: Life-Table Analysis of Surgical Treatment," The Annals of Thoracic Sur- gery, Vol. 27, No. 1, 1979, pp. 3-6. doi:10.1016/S0003-4975(10)62961-X

[6] C. F. Kittle, L. P. Faber, R. J. Jensik, et al., "Pulmonary Resection in Patients after Pneumonectomy,” The Annals of Thoracic Surgery, Vol. 40, No. 3, 1985, pp. 294-299. doi:10.1016/S0003-4975(10)60045-8

[7] P. Levasseur, J. F. Regnard, P. Icard, et al., "Cancer Surgery on a Single Residual Lung," European Journal of Cardio-Thoracic Surgery, Vol. 6, No. 12, 1992, pp. 639641. doi:10.1016/1010-7940(92)90187-3

[8] C. J. Westermann, H. A. van Swieten, A. Brutel de la Riviere, et al., "Pulmonary Resections after Pneumonectomy in Patients with Bronchogenic Carcinoma,” The Journal of Thoracic and Cardiovascular Surgery, Vol. 106, No. 5, 1993, pp. 868-873.

[9] N. Martini and M. Melamed, "Multiple Primary Lung Cancers," Journal of Thoracic and Cardiovascular Surgery, Vol. 70, No. 4, 1975, pp. 606-611.

[10] R. Abbey-Smith, B. K. Nigam, et al., "Second Primary Lung Cancer Carcinoma,” Thorax, Vol. 31, No. 5, 1976, pp. 507-516. doi:10.1136/thx.31.5.507

[11] R. J. Jensik, L. P. Faber and C. F. Kittle, "Survival Following Resection for Second Primary Bronchogenic Carcinoma," The Journal of Thoracic and Cardiovascular Surgery, Vol. 82, No. 5, 1981, pp. 658-663.

[12] Bitish Thoracic Society, "Guidelines on the Selection of Patients with Lung Cancer for Surgery,” Thorax, Vol. 56, No. 2, 2001, pp. 89-108. doi:10.1136/thorax.56.2.89

[13] G. Massard, J. M. Wihlm and G. Monard, "Surgical Management for Metachronous Bronchogenic Carcinoma after Pneumonectomy," The Journal of Thoracic and Cardiovascular Surgery, Vol. 109, No. 3, 1995, pp. 597-599. doi:10.1016/S0022-5223(95)70300-4 